Agric. Biol. Chem., 42 (6), $1201 \sim 1206,1978$

\title{
Etude de Quelques Propriétés de l'Aconitase de la Levure Saccharomycopsis lipolytica
}

\author{
B. Treton et H. Heslot \\ Institut National de la Recherche Agronomique. Institut National Agronomique- \\ 16. Rue Claude Bernard 75231 Paris Cédex 05, France
}

Received December 26, 1977

\begin{abstract}
Kinetic data are given on aconitase (EC 4.2.1.3.) of a strain of Saccharomycopsis lipolytica excreting citric acid. Various inhibitors were tested in vitro. Sigmoid inhibition curves (Hill coefficient $=4 \sim 6$ ) were observed with maleic and adipic acids. The effect of the inhibitors on excretion is discussed.
\end{abstract}

Certaines souches de la levure Saccharomycopsis lipolytica, poussant sur $n$-alcanes, excrètent des quantités importantes d'acides citrique et isocitrique. Tabuchi et Hara, ${ }^{11}$ Akiyama et al. $^{23}$ ont étudié l'effet du fluoroacétate sur cette excrétion en vue de diminuer le pourcentage d'isocitrate. En effet, la citrate synthétase transforme, in vivo, le fluoroacétate en fluorocitrate, ce dernier étant un inhibiteur classique de l'aconitase. Les auteurs ont effectivement observé une diminution du pourcentage d'isocitrate en présence de fluoroacétate à $0,1 \%$.

Akiyama et $a l^{2 /}$ sont parvenus à obtenir une souche mutante de $S$. lipolytica où l'activité aconitase est réduite à $1 \%$ de celle de la souche de départ. Cette souche mutante excrète un mélange composé de $97 \%$ d'acide citrique et $3 \%$ d'acide isocitrique.

Nous avons étudié les caractéristiques cinétiques de l'aconitase d'une autre souche de $S$. lipolytica, recherché des composés inhibiteurs in vitro et examiné si ces substances étaient susceptibles d'agir in vivo en améliorant la spécificité de l'excrétion.

\section{MATERIEL ET METHODES}

Microorganismes et méthode de culture. On a étudié la souche WT de Saccharomycopsis lipolytica dérivée de la souche IFP 29 après plusieurs sousclônages et sélection.

La composition par litre du milieu YEA de conservation des souches était la suivante : extrait de levure
$5 \mathrm{~g}$, glucose $20 \mathrm{~g}$, Difco bacto agar $20 \mathrm{~g}$.

Les cultures liquides ont été effectuées en milieu synthétique MMT de composition suivante (par litre): mélange de sels minéraux ${ }^{3)} 100 \mathrm{ml}$, thiamine $100 / \mathrm{g}$, glucose $10 \mathrm{~g}$; la source d'azote est constituée de sulfate d'ammonium. Ce milieu de base a parfois subi des modifications, comme il sera indiqué dans le courant de l'exposé : remplacement de la source de carbone ou d'azote, adjonction de citrate ou de glutamate.

Les cultures, d'un volume de 1 litre, ont été réalisées dans des fioles coniques de 2 litres, à $30^{\circ} \mathrm{C}$ et sous agitation. Ensemencées à partir d'un tube stock, elles ont été récoltées en fin de phase exponentielle.

Dans certains cas, le milieu MMT a été solidifié par adjonction de bacto agar $(20 \mathrm{~g} /$ litre $)$ et réparti dans des boites de Pétri.

Il a été également effectué des cultures en fioles de Ferobach de 2 jitres contenant $200 \mathrm{ml}$ du milieu d'excrétion mis au point par la firme Ajinomoto, ${ }^{4)}$ additionné de thiamine ( $1 \mathrm{mg} / \mathrm{litre})$, de coupe paraffinique constituée d'un mélange d'alcanes de 13 à 18 atomes de carbone $(20 \mathrm{~g} /$ litre $)$ et de $\mathrm{CaCO}_{3}(20 \mathrm{~g} /$ litre $)$ pour éviter son acidification. Dans ce cas la durée des cultures était d'une semaine. Les adjonctions éventuelles ont été opérées au 3ème jour, c'est-à-dire après la croissance cellulaire.

Préparation des extraits. Les cellules ont été récoltées par centrifugation (centrifugeuse MSE) et lavées deux fois à l'eau distillée.

Le culot cellulaire, pesé, a été repris dans son poids de tampon de broyage, de composition suivante: TRIS $0,05 \mathrm{M}$ pH 7,5, Chlorhydrate de cystéine $10^{-3} \mathrm{M}$, glycérol $30 \%$ (en poids) et additionné de deux fois son poids de billes de verre (diamètre $0,45 \sim 0,55 \mathrm{~mm}$ ). Il a été alors placé dans une fiole de broyage et agité dans un broyeur Braun réfrigéré, 30 secondes à faible vitesse, puis $1 \mathrm{~min}$ à vitesse maximum. Le broyat a été centrifugé 45 min à $57,000 \mathrm{~g}$ (centrifugeuse Spinco 
Beckman Ultracentrifuge). Le surnageant constitue l'extrait brut qui a été utilisé dans les dosages enzymatiques.

Mesure de l'activité aconitase. Elle a été dosée à $25^{\circ} \mathrm{C}$ par la méthode de Racker, ${ }^{5)}$ soit à partir de citrate trisodique $(0,1 \mathrm{M})$ soit à partir d'isocitrate trisodique (forme DL, concentration de la forme active $0,01 \mathrm{M}$ ) et suivie en continu dans un spectrophotomètre Beckman DK $2 \mathrm{~A}$ contre un témoin dépourvu de substrat. La quantité d'extrait adéquate contient environ 100 à $125 \mu \mathrm{g}$ de protéines. Celles-ci ont été mesurées par la méthode de Lowry et al. ${ }^{8)}$

Mesture de l'excrétion. Le surnageant des cultures en fiole de Fernbach a été acidifié pour éliminer le carbonate. On y a dosé le citrate par la méthode de Marier et Boulet ${ }^{\text {) }}$ et l'isocitrate par la méthode enzymatique d'Ochoa. ${ }^{8}$ La méthode de Marier et Boulet donnant globalement la concentration de citrate plus un quart de celle de l'isocitrate, il a été effectué la correction nécessaire.

\section{RESULTATS}

\section{Etudes cinétiques}

L'aconitase se conserve bien dans les cellules congelées. Elle est moins stable dans l'extrait brut. En deux jours (à $4^{\circ} \mathrm{C}$ ) l'extrait perd $30 \%$ d'activité, sauf en présence de fluorure de phényl-méthyl sulfonate $1 \mathrm{~mm}$. L'adjonction d'ions ferreux n'est pas nécessaire pour activer l'enzyme.

La courbe de saturation de l'aconitase par l'isocitrate est michaelienne, avec $K_{M}=9,5$. $10^{-5} \mathrm{M}$.

Les courbes obtenues avec le citrate sont moins simples. En tampon TRIS $0,1 \mathrm{M}$ on obtient deux pentes en coordonnées inverses, conduisant à deux valeurs de $\mathrm{K}_{\mathrm{M}}$, respective-

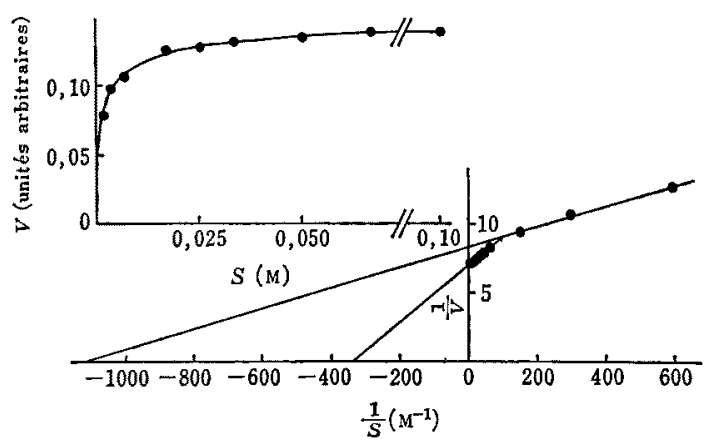

FIG. 1. Constante de Michaelis-Menten de l'aconitase pour le citrate (tampon TRIS $0,1 \mathrm{M}, \mathrm{pH} 7,5$ ). ment $0,9 \cdot 10^{-3} \mathrm{M}$ et $2,9 \cdot 10^{-3} \mathrm{M}$ (Fig. 1). En tampon phosphate $0,05 \mathrm{M} \mathrm{pH} \mathrm{7,0,} \mathrm{un} \mathrm{extrait}$ frais donne une courbe de saturation michaélienne avec $K_{M}=2,6 \cdot 10^{-3}$. Toutefois si cet extrait est conservé au congélateur pendant 24 heures, on obtient à nouveau deux pentes avec $\mathrm{K}_{\mathrm{M}}=2,3 \cdot 10^{-3} \mathrm{M}$ et $8,9 \cdot 10^{-3} \mathrm{M}$.

\section{Effet $d u p H$}

L'activité de l'aconitase a été mesurée dans

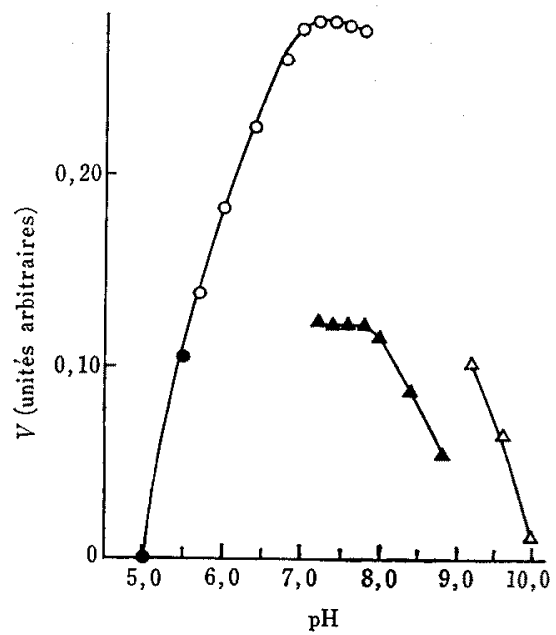

Fig. 2. Effet du pH sur l'activité de l'aconitase (substrat: isocitrate).

Tampons utilisés: $(-)$ acétate, $(O-O)$ phosphate, (A-A) Tris, $(\Delta-\Delta)$ carbonate. Tous sont en concentration $0,1 \mathrm{M}$.

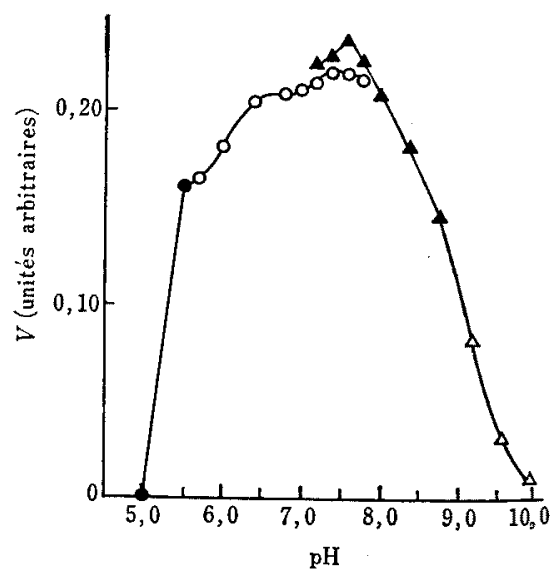

FIG. 3. Effet du pH sur l'activité de l'aconitase (substrat citrate).

(-) acétate, (O-O) phosphate, (A-A) Tris, $(\square-\square)$ carbonate. Tous sont en concentration $0,1 \mathrm{M}$. 
différents tampons de concentration $0,1 \mathrm{M}$ couvrant une large gamme de $\mathrm{pH}$. Les figures 2 et 3 rendent compte des résultats.

Quand le substrat est l'isocitrate, l'activité est maximum entre $\mathrm{pH} 7,2$ et 7,8 en tampon TRIS, ainsi qu'en tampon phosphate. Cependant l'activité au pH optimum est nettement plus élevée en tampon phosphate qu'en tampon TRIS $(\times 2,3)$.

Quand le substrat est le citrate, on note un maximum d'activité vers $\mathrm{pH} \mathrm{7,4}$ en tampon phosphate, pH 7,6 en tampon TRIS. Les activités sont très voisines dans ces deux tampons. En tampon phosphate, on observe vers $\mathrm{pH} 6,6$ un épaulement léger mais reproductible.

\section{Inhibiteurs}

Nous avons étudié plusieurs inhibiteurs potentiels qu'on peut classer en agents chélatants (acide quinaldique, O-phénantroline) et acides carboxyliques (fluorocitrate, maléate, adipate).

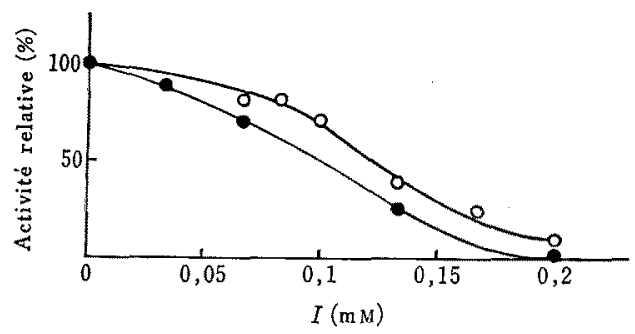

FIg. 4. Effet de l'acide quinaldique sur l'activité de l'aconitase.

Substrat: $(-)$ ) citrate, $\left(\mathrm{O}-O^{-}\right)$isocitrate. Tampon TRIS $0,1 \mathrm{M}, \mathrm{pH} 7,5$.

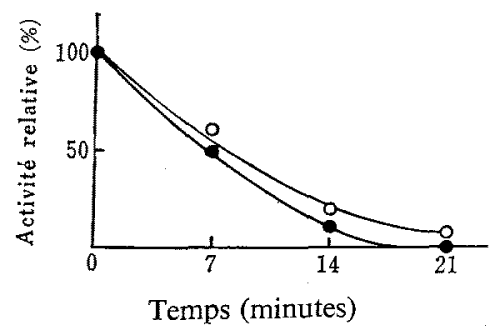

Fig. 5. Effet de L'O-phénanthroline sur l'activité de l'aconitase.

Substrat: (-) citrate, $(\mathrm{O}-\mathrm{O})$ isocitrate. Tampon TRIS $0,1 \mathrm{M}, \mathrm{pH} 7,5$. O-phénanthroline $0,167 \mathrm{~mm}$. a) Chélatants. L'incubation a eu lieu à $25^{\circ} \mathrm{C}$, dans le milieu réactionnel, avant adjonction du substrat. Les résultats obtenus avec l'acide quinaldique et l'O-phénanthroline sont représentés dans les figures 4 et 5 .

b) Acides carboxyliques. Le fluorocitrate est sans doute l'inhibiteur le plus connu de l'aconitase. La figure 6 montre :que l'inhibition est compétitive par rapport au citrate. La représentation de Dixon indique un $K_{i}$ de $3,5 \cdot 10^{-4} \mathrm{M}$.

Le maléate donne une courbe d'inhibition

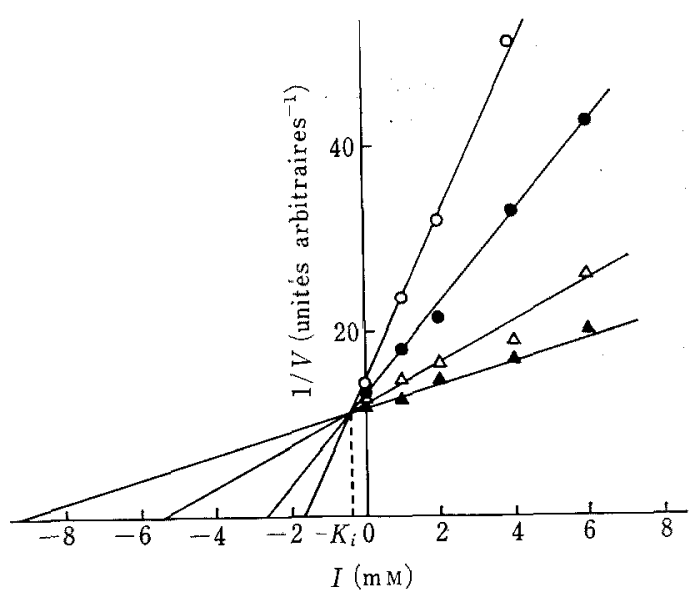

Fra. 6. Effet du fluorocitrate sur l'activité de l'aconitase: Représentation de Dixon.

Substrat: citrate $(\mathrm{O}-\mathrm{O}) 10 \mathrm{~mm},(--) 20 \mathrm{~mm}$,

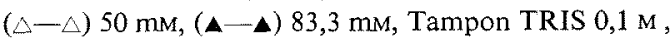
pH 7,5.

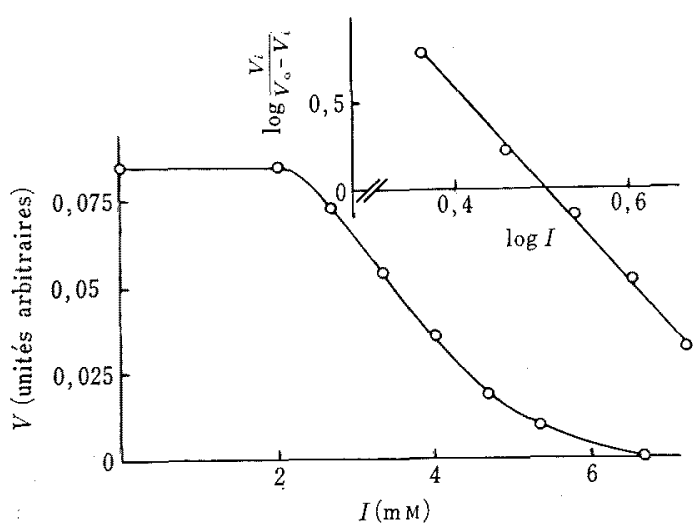

Fig. 7. Effet du maléate sur l'activité de l'aconitase (A) et représentation de Hill (B).

Substrat: isocitrate, Tampon TRIS $0,1 \mathrm{M}, \mathrm{pH} 7,5$. 
sigmoïdale (Fig. 7A). Avec l'isocitrate comme substrat, la représentation de Hill donne une droite de pente $n=5,60$ (Fig. 7B) et deux droites ( $n=0,96$ et $n=4,94$ ) dans le cas du citrate.

L'adipate donne aussi une courbe d'inhibition sigmoïdale (Fig. 8A). Dans le cas du citrate, la représentation de Hill donne deux droites de pentes respectives $n=1,14$ et 4,9 . Pour l'isocitrate, $n=4,24$ (Fig. 8 B).

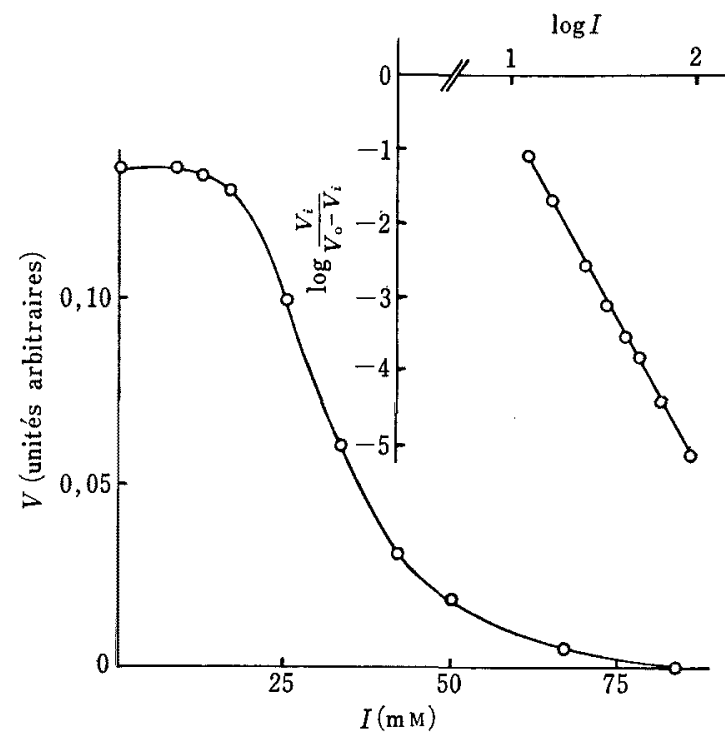

FIG. 8. Effet de l'adipate sur l'activité aconitase (A) et représentation de Hill (B).

Substrat: isocitrate, Tampon TRIS $0,1 \mathrm{M}, \mathrm{pH} 7,5$.

\section{Activité spécifique de l'aconitase dans différents} milieux

L'activité de l'aconitase a été mesurée sur des cellules récoltées en fin de phase exponentielle. Les résultats sont indiqués dans le Tableau I.

Les différences d'activité spécifique d'un substrat carboné à un autre indiquent une sensibilité de la souche de $S$. lipolytica à la répression catabolique.

L'activité spécifique est très élevée quand la source de carbone est du citrate. Elle est encore significativement supérieure au témoin MMT glucose quand le citrate n'est pas la source unique de carbone. La répression catabolique ne semble donc pas être seule en cause, et il n'est pas exclu que le citrate
Tableau I. Effet de la Source de Carbone ET DE Diverses ADJONCTIONS SUR L'ACTIVITÉ SPÉCIFIQUE

Le substrat utilisé était le citrate. Les activités sont exprimées en $\mu$ moles de cis-aconitate formé par minute et par mg de protéines.

\begin{tabular}{|c|c|c|}
\hline Milieu & $\begin{array}{l}\text { Activité } \\
\text { spécifique }\end{array}$ & $\begin{array}{c}\% \text { de la valeur } \\
\text { observée en } \\
\text { milieu MMT } \\
\text { glucose }\end{array}$ \\
\hline MMT glucose $10 \mathrm{~g} /$ litre & 0,065 & 100 \\
\hline $\begin{array}{l}\text { MMT glucose } 10 \mathrm{~g} / \text { litre } \\
\quad+\text { glutamate } 2 \mathrm{~g} / \text { litre }\end{array}$ & 0,069 & 106 \\
\hline $\begin{array}{l}\text { MMT glucose } 10 \mathrm{~g} / \text { litre } \\
\text { + glutamate } 11 \mathrm{~g} / \text { litre } \\
\text { (source d'azote) }\end{array}$ & 0,099 & 152 \\
\hline MMT glycérol $10 \mathrm{~g} /$ litre & 0,049 & 75 \\
\hline MMT acétate $10 \mathrm{~g} /$ litre & 0,224 & 345 \\
\hline MMT citrate $10 \mathrm{~g} /$ litre & 0,728 & 1120 \\
\hline $\begin{array}{c}\text { MMT glucose } 10 \mathrm{~g} / \text { litre } \\
+ \text { citrate } \\
16 \mathrm{~g} / \text { litre }\end{array}$ & 0,181 & 278 \\
\hline
\end{tabular}

agisse comme inducteur de l'aconitase, comme cela a été signalé chez $B$. subtilis. ${ }^{9} \quad$ Ces faits sont en accord avec les observations que Suzuki et $a l^{\left.1{ }^{10}\right)}$ rapportent dans le courant de leur étude sur l'effet inducteur du fluorocitrate. Cet effet est encore beaucoup plus net que celui du citrate. Il avait déjà été mentionné chez Escherichia coli. ${ }^{11}$ Nous ne l'avons pas recherché chez notre souche.

Le glutamate n'exerce pas d'effet répresseur sur l'aconitase. Employé comme source unique d'azote, il donne même une activité spécifique légèrement supérieure au témoin MMT glucose.

\section{Essais d'inhibition de l'aconitase in vivo}

Nous avons cherché à inhiber l'aconitase de la souche sauvage en introduisant dans le milieu d'excrétion un composé reconnu inhibiteur de cet enzyme in vitro.

Nous avons d'abord effectué des essais préliminaires pour déterminer la concentration à utiliser. Nous avons ensuite procédé à des tests d'excrétion en fiole. Dans les tests d'excrétion, l'inhibiteur a été ajouté en fin de croissance. Les résultats sont indiqués dans le Tableau II. L'O-phénanthroline inhibe totalement l'excrétion et provoque la mort des cellules. Par contre ni l'adipate, ni le maléate ne modifient la quantité totale des acides 
Tableau II. Inhibiteurs de L'aconitase-Toxicité et Effet sur L'Excrétion

Les essais préliminaires portant sur la toxicité ont été faits en milieu MMT solide dans le cas de l'Ophénanthroline et du quinaldate, en milieu MMT solide supplémenté ou non par du glutamate (100 mg/liter), en milieu YEA solide et en milieu MMT liquide dans le cas du fuoroacétate, en milieu MMT liquide dans les autres cas.

\begin{tabular}{|c|c|c|c|c|}
\hline \multirow{2}{*}{ Inhibiteur } & \multicolumn{2}{|c|}{ Effets sur la croissance } & \multicolumn{2}{|c|}{ Effets sur l'excrétion } \\
\hline & $\begin{array}{l}\text { Concentration } \\
\text { essayée }\end{array}$ & Résultats & $\begin{array}{l}\text { Concentration } \\
\text { essayée }\end{array}$ & Résultats \\
\hline O-Phénanthroline & $10^{-7}$ à $10^{-4} \mathrm{M}$ & $\begin{array}{l}\text { inhibition partielle } \\
\text { à } 5.10^{-5} \mathrm{M} \\
\text { totale à } 10^{-4} \mathrm{M}\end{array}$ & $10^{-6} \mathrm{M}$ & blocage de l'excrétion \\
\hline Quinaldate & $10^{-7}$ à $10^{-4} \mathrm{M}$ & néant & $10^{-5} \mathrm{M}$ & $\begin{array}{l}\text { amélioration légère } \\
\text { de l'excrétion-Pas } \\
\text { d'effet sur la spécificité }\end{array}$ \\
\hline Fluoroacétate & 0,01 à $10 \%$ & $\begin{array}{c}\text { inhibition partielle } \\
\text { de } 0,01 \text { à } 5 \%\end{array}$ & - & - \\
\hline Adipate & $1 \%$ & néant & $1 \%$ & néant \\
\hline maléate & 0,03 à $1 \%$ & néant & $1 \%$ & néant \\
\hline
\end{tabular}

citrique et isocitrique excrétés et n'exercent d'influence sur le pourcentage d'isocitrate. En ce qui concerne le fluoroacétate, on note que la souche y est très résistante. Nous n'avons pas étudié l'influence de ce composé sur l'excrétion.

\section{DISCUSSION}

La cinétique michaélienne trouvée pour le substrat isocitrate ne pose pas de problème et le $\mathrm{K}_{\mathrm{M}}$ correspondant est voisin des valeurs signalées pour l'aconitase d'autres organismes.

Par contre, l'interprétation des courbes de saturation de l'enzyme par le citrate est moins aisée. En effet on observe deux droites en coordonnées inverses, ce qui pourrait faire penser à une deuxième activité, par exemple une citrate déshydratase, du type de celle décrite chez Aspergillus. ${ }^{12)}$ Cependant le fait qu'en tampon phosphate, un extrait frais ne donne qu'une seule valeur de $K_{M}$, alors que le même extrait, conservé 24 heures au congélateur en donne deux, conduit à rechercher une autre explication. On peut envisager l'une des possibilités suivantes:

a) Dans certains tampons, l'aconitase existerait sous forme de monomères et sous forme de dimères (ou multimères). Ces derniers ne pourraient plus transformer le substrat isocitrate, mais seraient encore parti- ellement actifs sur le citrate. Cette hypothèse nous a été suggérée par les observations de Zweerinck $^{13)}$ chez plusieurs espèces de bactéries.

b) Il se peut que le citrate à forte concentration exerce un effet activateur ou protecteur sur l'aconitase, placée dans un tampon défavorable ou ayant subi un début de dénaturation.

c) Il est également possible que la réversibilité de la réaction intervienne. A forte concentration de citrate, il se peut que la réaction d'isomérisation soit favorisée, produisant directement de l'isocitrate, alors déshydraté en $c i s$-aconitate, qui vient s'ajouter à celui que produit la déshydratation directe du citrate.

Nous avons vu que l'aconitase de $S$. lipolytica est sensible à des inhibiteurs bien connus tels que le fluorocitrate et les chélatants du fer.

Les effets inhibiteurs des deux chélatants O-phénanthroline et acide quinaldique sont différents. Le premier doit incuber avec l'enzyme pour l'inhiber efficacement, alors que l'action du second est immédiate. Il se peut que l'effet inhibiteur de l'acide quinaldique ne soit pas dû uniquement à sa capacité à chélater le fer. Chez B. subtilis, Fortnagel et Freese ${ }^{14}$ ) ont montré qu'au contraire c'est l'O-phénanthroline qui est l'inhibiteur le plus puissant.

Contrairement aux observations de Villafranca $^{15\rangle}$ sur l'enzyme de coeur de porc, le 
maléate est inhibiteur de l'aconitase chez $S$. lipolytica. Cette inhibition présente des caractéristiques similaires à celle exercée par l'adipate. La valeur élevée des coefficients de Hill nous conduit à penser qu'il peut s'agir d'une agglutination de l'enzyme favorisée par ces composés. Gawron et al ${ }^{16)}$ ont en effet signalé que chez de nombreux organismes, les molécules d'aconitase s'agglomèrent facilement; Suzuki et al. ${ }^{17)}$ l'ont également constaté lors de la purification de l'aconitase d'une autre souche de S. lipolytica.

Nous n'avons pas réussi à diminuer le pourcentage d'isocitrate en ajoutant au milieu de fermentation un inhibiteur de l'aconitase.

Dans certains cas (adipate, maléate), la pénétration de l'inhibiteur dans les cellules est douteuse. Dans d'autres cas (O-phénanthroline), l'inhibiteur s'est révélé trop toxique, peut-être parce qu'il affecte d'autres activités enzymatiques essentielles, sensibles à des concentrations inférieures à celles qui inhibent l'aconitase. Quant à l'acide quinaldique, il n'améliore pas la spécificité de l'excrétion, du moins à la concentration essayée.

Le fluoroacétate n'est inhibiteur qu'à des concentrations élevées. Cette résistance de notre souche de $S$. lipolytica peut s'expliquer par une mauvaise pénétration du fluoroacétate ou par un faible taux de transformation en fluoroacétylcoenzyme A. puis en fluorocitrate. Ce dernier s'est montré un bon inhibiteur de l'aconitase in vitro.

Nous n'avons pas recherché l'effet du fluoroacétate sur l'excrétion. Mais chez $S$. lipolytica, Tabuchi et Hara' ${ }^{1)}$ rapportent qu'en présence de fluoroacétate à $1 \%$, l'excrétion d'acide citrique sur glucose est très augmentée, tandis que sur $n$-paraffines, elle diminue légèrement. Ces auteurs interprètent ces résultats comme une indication du mode de régénéra- tion de l'oxaloacétate qui nécessite le fonctionnement du shunt glyoxylique dans le cas des $n$-paraffines, et par conséquent une forte activité de l'aconitase, tandis que sur glucose ce n'est pas nécessaire. Par contre Akiyama et $a l .^{2)}$ observent une diminution du pourcentage d'isocitrate-qui passe de $40 \%$ à $15 \%$ mais une légère augmentation de la production totale, en présence de fluoroacétate à $0,1 \%$.

Remerciements. Nous remercions Mlle M.T. LE DALL pour son excellente assistance technique.

\section{REFERENCES}

1) T. Tabuchi et S. Hara, Nippon Nôgeikagaku Kaishi, 48, 417 (1974).

2) S. Akiyama, T. Suzuki, Y. Sumino, Y. Nakao et H. Fukuda, Proc. IV IFS: Ferment. Technol. Today, 1972, 613.

3) U. Leupold, Schweiz. Z. Allg. Pathol. Bakteriol, 15, 1141 (1955).

4) Ajinomoto, Brevet japonais $n^{\circ} 13939,1968$.

5) E. Racker, Biochim. Biophys. Acta, 4, 211 (1950).

6) O. H. Lowry, N. J. Rosebrough, A. L. Farr et R. J. Randall, J. Biol. Chem., 193, 265 (1951).

7) J. R. Marier et M. Boulet, J. Dairy Sci., 41, 1683 (1958).

8) S. Ochoa, J. Biol. Chem., 174, 133 (1948).

9) M. Ohne, J. Bacteriol., 117, 1295 (1974).

10) T. Suzuki, S. Akiyama, O. Yamazaki, K. Nara, Y. Nakao et H. Fukuda, Agric. Biol. Chem., 39, 97 (1975).

11) Y. Takahashi, J. Gen. Appl. Microbiol., 17, 479 (1971).

12) N. E. Neilson, J. Bacteriol., 7, 356 (1956).

13) H. J. Zweerinck, Dissert. Abstracts, 28, 2972B (1968).

14) P. Fortnagel et E. Freese, J. Biol. Chem., 243, 5289 (1968).

15) J. J. Villafranca, J. Biol. Chem., 249, 6149 (1974).

16) O. Gawron Sr., M. C. Kennedy et R. A. Rauner, Biochem. J., 143, 717 (1974).

17) T. Suzuki, O. Yamazaki, K. Nara, S. Akiyama, Y. Nakao et H. Fukuda, J. Biochem., 77, 367 (1975). 